\title{
Simple equipment for studying the injection molding process
}

\author{
Adelina Hrițuc* \\ "Gheorghe Asachi” Technical University of Iasi, Blvd. 59A, 700050 Iasi, Romania
}

\begin{abstract}
The injection molding process is largely applied to obtain plastic parts. The problem of finding a simple equipment able to allow the study of the injection process was addressed in the research presented in this paper. Aiming to solve the problem, the main requests valid for the proposed equipment were formulated. Considering some possible versions of the equipment subassemblies, the ideas diagram method and the method of imposed decision were applied to select the most convenient version of the injection equipment. As a result, a constructive solution for a simple injection molding equipment that could be used to develop some experimental researches was identified.
\end{abstract}

\section{Introduction}

Plastics are a mixture of two or more polymers with one or more additives. They are macromolecular materials - such as high molecular weight chemicals, obtained by polymerization. The plastic materials are of two types: thermoplastics and thermorigides. The thermoplastics are characterized in that they exhibit the character of reversible transformation. They are transformed into products under the action of heat, requiring cooling to keep their shape. They do not undergo chemical transformations during the formation of the parts, and thus they can be melted again. The thermorigides are characterized by the fact that under the action of heat and pressure during processing, they undergo irreversible chemical transformations [1]. Due to their own properties, the plastics can replace the deficient metallic materials, making the manufacture more economical. Plastic materials are especially used when parts must be obtained with less material consumption and at a lower cost. They are used in areas where it is unprofitable to use metallic materials, being yet more resistant to atmospheric agents in particular.

The polymers are considered as special materials (due to their good mechanical and thermal resistance, corrosion resistance, and low density, and good machinability, low electrical and thermal conductivity). Essentially, they are used in conditions in which the so-called classical materials do not face. The plastics are usually lighter than the metallic material, have a much lower density, are easier to process, are resistant to atmospheric, chemical and aquatic agents, and are good insulators, sometimes transparent as glass, but unbreakable, resistant to bacteria and moisture. They ensure a high degree of material utilization, the possibility of obtaining parts of immiscible materials, as well as of some that

\footnotetext{
*Corresponding author: hrituc.adelina3295@yahoo.com
} 
could work in conditions of self-lubrication [2]. The injection, along with extrusion, blowing and calendering, thermoforming, vacuum forming, compression molding, and rotomolding are the main techniques for forming plastics.

The injection molding is a particularly complex process, consisting of a large number of phases and phenomena that develop both simultaneously and successively, until a good quality injection piece is obtained.

The compaction of the material is an important aspect taken into account at the injection process, the material being "guided" through the injection barrel and forced to take the proper form. The material contraction at solidification must be also known, since it influences the dimensions of the final piece [3].

Over time, the plastic injection process has been studied from several distinct perspectives. For example, the researchers had preoccupations to identify the influence of certain manufacturing process input factors on the properties of the obtained parts. Thus, M. Sardarian et al. developed a research concerning the influence of temperature and pressure in the injection process to ensure it optimal development [4]. Their results showed that the increase of the injection temperature and pressure could determine the formation of voids affecting the properties of the molded parts.

Matarrese et al. investigated the energy consumed in the process of injection molding and the influence exerted by this process on the environment. The estimation of energy consumption during the injection process could improve the evaluation of the overall environmental impact of the manufactured products [5].

The researchers also addressed other problems such as, for example the influence exerted by the friction phenomena on the injection molding process. The experimental results proved significant interactions between the mold surface texture and the parameters of the microinjection molding, such are the mold temperature and the pressure [6].

By analyzing the factors that influence the manufacturing process of plastic parts using the process of injection molding, it is possible to design and achieve a simple equipment to experimentally investigate the influence of some process input factors on the quality of the parts obtained by plastic injection.

\section{Initial premises}

The injection molding is the process by which a macromolecular material brought in a plastic state at a certain temperature, called the softening temperature, is injected under high pressure into the mold cavity. At the end of the pressing action, the plastic retains the shape

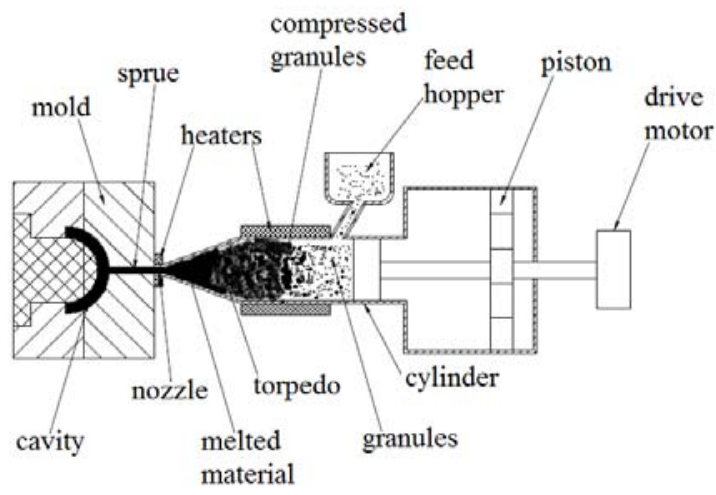

Fig. 1. Injection molding process. of the mold in which it was injected, and after its cooling, it can be removed from the mold.

The injection process has a cyclic character, involving stages of material feeding, compressing, thermoplastifying, injecting, cooling and, finally, opening of the mold and evacuating of the injected piece. Subsequently, a new injection cycle develops. The thermoplastic macromolecular compounds modify slightly their physical properties between the solidification and the melting temperature. The injection is based on the properties of the plastic 
material between the softening and flowing temperatures. The process has significant advantages, especially due to its high productivity. Thus, there are the possibilities of mechanization and automation of the process, of manufacturing complex and diverse parts, without requiring any overworking. One of the interest results is the relatively low cost per product. The injection process is considered as economical in the conditions of producing a series fabrication to cushion the costs of the mold [7].

The injection process is influenced by some factors that must be taken into consideration when designing and developing the manufacturing process. Such factors could be the temperature of both the material and the mold, the pressure, the size of the hole from the nozzle, of the injection barrel and of the sprue, the properties of the plastic material, the piston speed during the material pressing, the size of the torpedo, of the zone where the material is homogenized (Figure 1) etc.

In the case of designing an injection equipment, it is important to study how the process develops from the introducing the plastic granules to the removal of the part from the mold. The processes of the granules melting and of homogenization of the melted material must be correctly achieved. Other aspects that must be taken into consideration concern the way in which the melted material reaches the nest and fills the entire mold cavity, the air is released outside and the material solidifies. At the same time, the speed at which the material is pushes, the pressure exerted on the material, the length of the injection barrel, the speed at which the process takes place and the temperature of the mold must be adequately planned. The diameter of the injection cylinder is an important factor in material plasticizing and flowing. The injection volume and the plasticizing capacity are other significant factors whose values must be considered.

Essential characteristics of interest in using the injection equipment could be the way of plastic heating, as well as the pressure and the speed of injection and of filling the mold nest and the time required for the material solidifying process.

\section{Functional requirements of the equipment}

The equipment will be designed by considering the basis of functional, manufacturing and constraining requirements that limit the design and influence the constructive-functional characteristics of the equipment, as well as the materials used in its achievement. Such requirements could be:

- The equipment is limited to a certain overall size;

Table 1. Functional requirements and design parameters.

\begin{tabular}{|c|c|c|c|c|c|c|c|}
\hline$D P$ & $\vec{\Omega}$ & 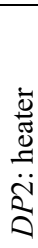 & 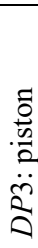 & 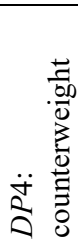 & $\begin{array}{l}\frac{0}{0} \\
\tilde{D} \\
\overline{0} \\
\ddot{\check{n}} \\
\frac{a}{\sigma}\end{array}$ & 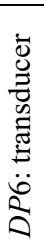 & 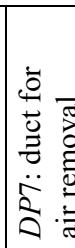 \\
\hline \begin{tabular}{|l|}
$F R 1$ : Introduce the granules \\
\end{tabular} & $\mathrm{X}$ & & & & & & \\
\hline FR2: Melt the granules melting & & $\mathrm{X}$ & & & & & \\
\hline FR3: Advance the granules & & & $\mathrm{X}$ & & & & \\
\hline FR4: Activate the system & & & & $\mathrm{X}$ & & & \\
\hline FR5: Homogenize the material & & & & & $\mathrm{X}$ & & \\
\hline $\begin{array}{l}\text { FR6: Highlight the temperature } \\
\text { value }\end{array}$ & & & & & & $\mathrm{X}$ & \\
\hline FR7: Ensure filling of cavity & & & & & & & $\mathrm{X}$ \\
\hline
\end{tabular}

- The equipment must be handled by one person;

- The equipment must be achieved by using the manufacturing and assembling facilities existing in a determined department;

- It is necessary to minimize the number of parts to be purchased;

- The equipment could be adapted on an existing machine tool from the department;

- The equipment can be made without using expensive or difficult-to-machine 
materials;

- The equipment could include simple subassemblies.

A smaller number of requirements were selected as functional requirements $(F R)$, in accordance with the principles of the axiomatic design $[8,9]$ and they were presented in Table 1. In this table, one could notice also the design parameters DP that correspond to each functional requirement.

\section{Outlining solutions for research equipment using the diagram of idea}

An ideas diagram was used to highlight the distinct possibilities of designing the equipment. The distinct versions of the equipment subassemblies must be taken into account.

The graphic representation corresponding to the ideas diagram was developed by identifying firstly the necessary components/ subassemblies of the equipment and including these components in rectangles placed along a horizontal line. Subsequently, below each of these components, along a vertical line, the distinct versions of the components were highlighted $[8,9]$.

Since the complete form of the ideas diagram is very complex, in figure 2 only a simplified version of the ideas diagram was included.

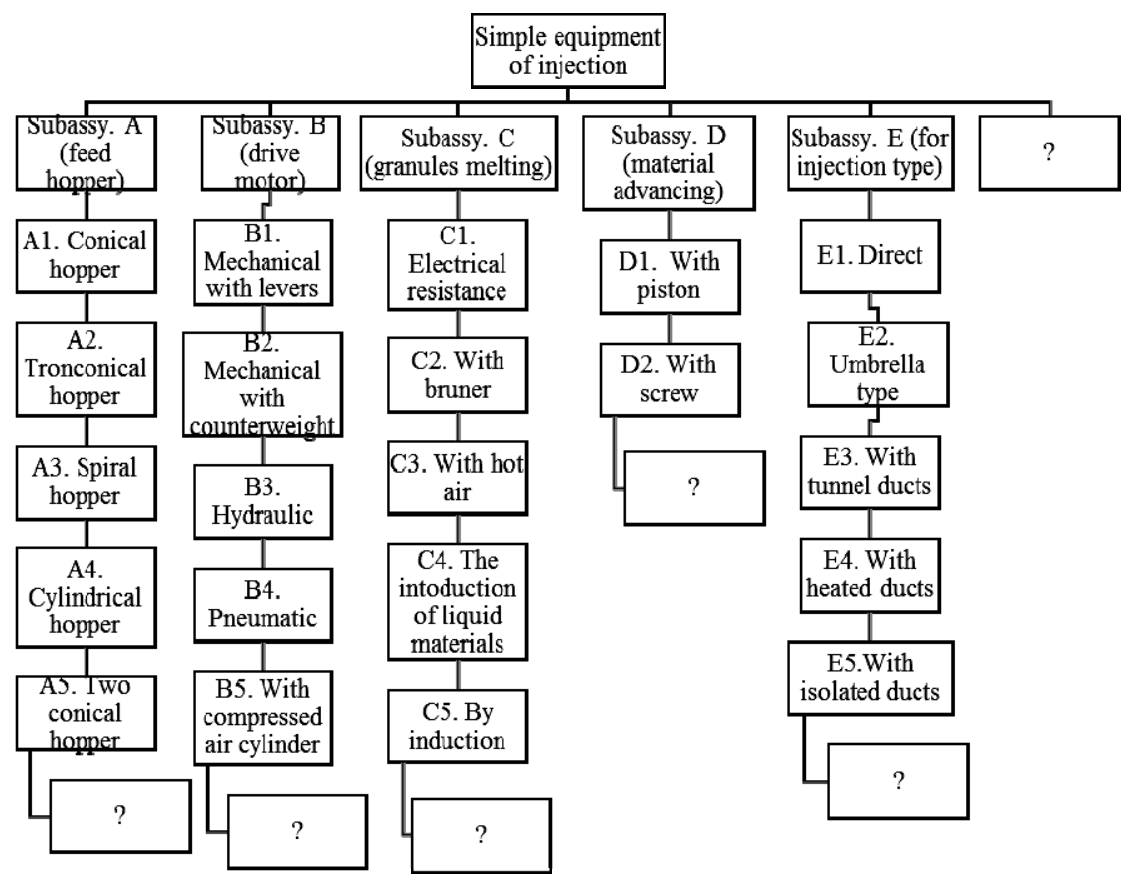

Fig. 2. Ideas diagram thought for the simple injection equipment.

\section{Choosing a solution for the equipment using the method of imposed decision}

Based on the ideas diagram, three distinct constructive solutions appreciated as interesting were identified (Figure 3). In accordance with the symbols used in figure 2, the selected 
Table 2. Values of the importance coefficients for each evaluation criterion.

\begin{tabular}{|l|l|l|l|l|l|l|l|l|l|l|l|l|l|}
\hline \multirow{2}{*}{ Considered criteria } & \multicolumn{7}{|c|}{ Comparison number } & $\begin{array}{l}\text { Coefficient of } \\
\text { importance }\end{array}$ \\
\cline { 2 - 12 } & 1 & 2 & 3 & 4 & 5 & 6 & 7 & 8 & 9 & 10 & & \\
\hline A- cost & 1 & 0 & 0.5 & 0.5 & & & & & & & 2 & 0.2 \\
\hline B- possibility of designing & 0 & & & & 0 & 0.5 & 0 & & & & 0.5 & 0.05 \\
\hline C- possibility of researching & & 1 & & & 1 & & & 1 & 1 & & 4 & 0.4 \\
\hline D- practical, ease of handling & & & 0.5 & & & 0.5 & & 0 & & 0.5 & 1.5 & 0.15 \\
\hline E- possibility of automation & & & & 0.5 & & & 1 & & 0 & 0.5 & 2 & 0.2 \\
\hline
\end{tabular}

solutions were $A 1 B 1 C 1 D 2 E 1, A 2 B 3 C 2 D 1 E 3$ and $A 1 B 2 C 1 D 1 E 1$. The intention of practical achieving of one of these solutions was formulated. To select the most convenient solution among the three identified ones, the method of the imposed solution was applied $[9,10]$.

The selection criteria are firstly hierarchized according to them coefficients of importance that denotes the ratio of the weight of each criterion to the number of comparisons of two by two criteria. Considering the values of the coefficient of importance established for the criteria, the order of the criteria is: $C-A-E-D-B$. In the next stage, the values of the coefficients of importance for each proposed solution were calculated using each criterion and comparing also two by two from the proposed solutions $a, b$ and $c$. The results of applying this stage of the imposed decision method were synthetically presented in Table 3. In the last stage, the value numbers for each solution were determined, based on the previously calculated values of the coefficients of importance established for each criterion and the coefficient of importance of each solution by applying a certain criterion:

$$
\begin{aligned}
& N v a=0.2 \cdot 0.166+0.05 \cdot 0.333+0.4 \cdot 0.166+0.15 \cdot 0.5+0.2 \cdot 0.5=0.294 \\
& N v b=0.2 \cdot 0.166+0.05 \cdot 0.166+0.4 \cdot 0.166+0.15 \cdot 0+0.2 \cdot 0.5=0.207 \\
& N v c=0.2 \cdot 0.666+0.05 \cdot 0.5+0.4 \bullet 0.666+0.15 \bullet 0.5+0.2 \cdot 0=0.499
\end{aligned}
$$

Using the method of the imposed decision and on the base of the coefficients of importance attributed to each solution, one established that the most convenient one is the solution $c(A 1 B 2 C 1 D 1 E 1)$. The equipment that corresponds to the solution $c$ could be adapted on a machine tool existing in the department.

\begin{tabular}{|c|c|c|c|c|c|c|c|c|c|c|c|c|c|c|c|c|c|}
\hline \multirow{3}{*}{ 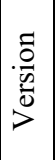 } & \multicolumn{5}{|c|}{ Criterion $A$} & & \multicolumn{5}{|c|}{ Criterion $B$} & & \multicolumn{5}{|c|}{ Criterion $C$} \\
\hline & \multicolumn{3}{|c|}{ Comparison } & \multirow[t]{2}{*}{$\Sigma$} & \multirow{2}{*}{$\begin{array}{c}\text { Coeff. } \\
\text { of } \\
\text { imp. }\end{array}$} & \multirow{2}{*}{$\begin{array}{l}\overline{0} \\
\bar{n} \\
\overline{0} \\
\\
>\end{array}$} & \multicolumn{3}{|c|}{ Comparison } & \multirow[t]{2}{*}{$\Sigma$} & \multirow{2}{*}{$\begin{array}{c}\text { Coeff. } \\
\text { of } \\
\text { imp. }\end{array}$} & \multirow{2}{*}{ 迹 } & \multicolumn{3}{|c|}{ Comparison } & \multirow[t]{2}{*}{$\Sigma$} & \multirow{2}{*}{$\begin{array}{c}\text { Coeff. } \\
\text { of } \\
\text { imp. }\end{array}$} \\
\hline & 1 & 2 & 3 & & & & 1 & 2 & 3 & & & & 1 & 2 & 3 & & \\
\hline$a$ & 0.5 & 0 & & 0.5 & 0.166 & $a$ & 0.5 & 0.5 & & 1 & 0.333 & $a$ & 0.5 & 0 & & 0.5 & 0.166 \\
\hline$b$ & 0.5 & & 0 & 0.5 & 0.166 & $b$ & 0.5 & & 0 & 0.5 & 0.166 & $b$ & 0.5 & & 0 & 0.5 & 0.166 \\
\hline $\bar{c}$ & & 1 & 1 & 2 & 0.666 & $c$ & & 0.5 & 1 & 1.5 & 0.5 & $c$ & & 1 & 1 & 2 & 0.666 \\
\hline \multirow{3}{*}{ 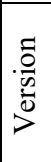 } & \multicolumn{5}{|c|}{ Criterion $D$} & & \multicolumn{5}{|c|}{ Criterion $E$} & & & & & & \\
\hline & \multicolumn{3}{|c|}{ Comparison } & $\Sigma$ & Coeff. &.$\frac{9}{4}$ & \multicolumn{3}{|c|}{ Comparison } & $\Sigma$ & Coeff. & & & & & & \\
\hline & 1 & 2 & 3 & & $\begin{array}{l}\text { of } \\
\text { imp. }\end{array}$ & $>$ & 1 & 2 & 3 & & $\begin{array}{l}\text { of } \\
\text { imp. }\end{array}$ & & & & & & \\
\hline$a$ & 1 & 0.5 & & 1.5 & 0.5 & $a$ & 1 & 0.5 & & 1.5 & 0.5 & & & & & & \\
\hline$b$ & 0 & & 0 & 0 & 0 & $b$ & & 0.5 & 1 & 1.5 & 0.5 & & & & & & \\
\hline$c$ & & 0.5 & 1 & 1.5 & 0.5 & $c$ & 0 & & 0 & 0 & 0 & & & & & & \\
\hline
\end{tabular}

Table 3. Determining the value numbers assigned to each version of solution by considering each of the criteria $j$ for an injection molding equipment. 

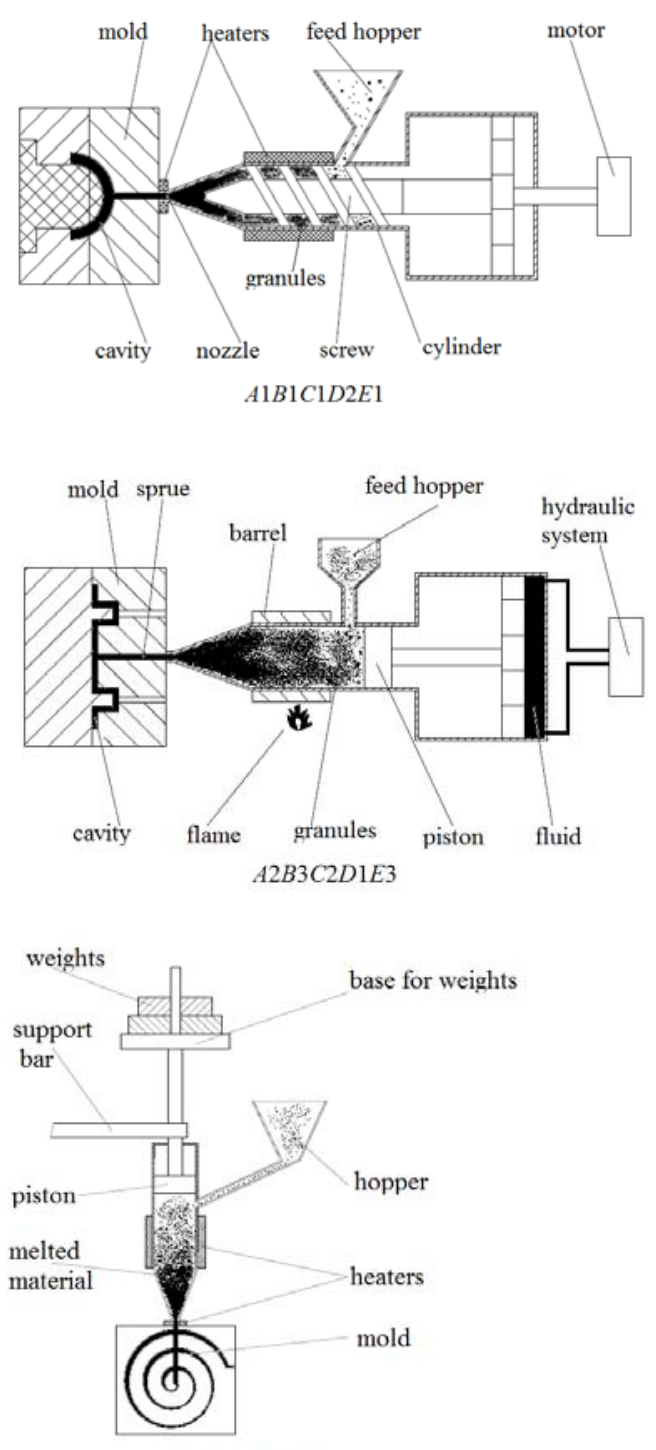

$A 1 B 2 C 1 D 1 E 1$

Fig. 3. Constructive solutions for the equipment.

\section{Conclusions}

One noticed that the injection process has triggered the interest of many researchers and has been studied from several distinct perspectives. By approaching of some theoretical considerations, one showed that it is possible to design and achieve a simple equipment to develop a scientific investigation of the plastics injection process. Applying some principles specific research of several research methods, namely axiomatic design method, the ideas diagram method, the method of the imposed decision, and one established the most convenient solution for a simple plastic injection equipment. In the future, there is the intention to materialize this equipment and to initiate some experimental researches aiming to show the influence exerted by some process input factors on the quality parameters of the injected parts.

\section{References}

1. A. Sover, Plastics manufacturing technologies (in Romanian), manuscript (2017)

2. C. Fetecău, Injection of plastics (in Romanian), (Ed. Did. si Ped., Bucureşti, 2007)

3. M. Ionescu, M. Ianculescu, I. V. Seres, I. Rosenthal, Design of molds for plastics injected products (in Romanian), (Publishing House, Bucureşti, 1987)

4. M. Sardarian, O. Mirzaee, A.

Habibolahzadeh, Ceram Int 43, 4785-4793 (2017)

5. P. Matarrese, A. Fontana, M. Sorlini, L. Diviani, A. Specht, A. Maggi, J Clean Prod 168, 1505-1512 (2017)

6. M. Sorgato, D. Masato, G. Lucchetta, Precis Eng 50, 440-448 (2017)

7. T. Iclănzan, Technology of plastics pressing and injection (in Romanian) (UPT Publishing House, Timişoara, 1992)

8. N.P. Suh., Axiomatic Design: Advances and Applications (Oxford University Press, New York, 2001)

9. L. Slătineanu, Industrial property (in Romanian) (Performantica, Iași, 2015)

10. V. Belous, Inventics (in Romanian) (Asachi Publishing House, Iași, 1992) 